This item was submitted to Loughborough's Research Repository by the author.

Items in Figshare are protected by copyright, with all rights reserved, unless otherwise indicated.

\title{
Influence of threshold variation on determining the properties of a polymer electrolyte fuel cell gas diffusion layer in X-ray nano-tomography
}

PLEASE CITE THE PUBLISHED VERSION

http://dx.doi.org/10.1016/j.ces.2009.12.019

PUBLISHER

(C) Elsevier

VERSION

AM (Accepted Manuscript)

LICENCE

CC BY-NC-ND 4.0

\section{REPOSITORY RECORD}

Ostadi, Hossein, Kyle Jiang, Pratap Rama, Yu Liu, Rui Chen, and Xiaoxian Zhang. 2011. "Influence of Threshold Variation on Determining the Properties of a Polymer Electrolyte Fuel Cell Gas Diffusion Layer in Xray Nano-tomography". figshare. https://hdl.handle.net/2134/8311. 
This item was submitted to Loughborough's Institutional Repository by the author and is made available under the following Creative Commons Licence conditions.

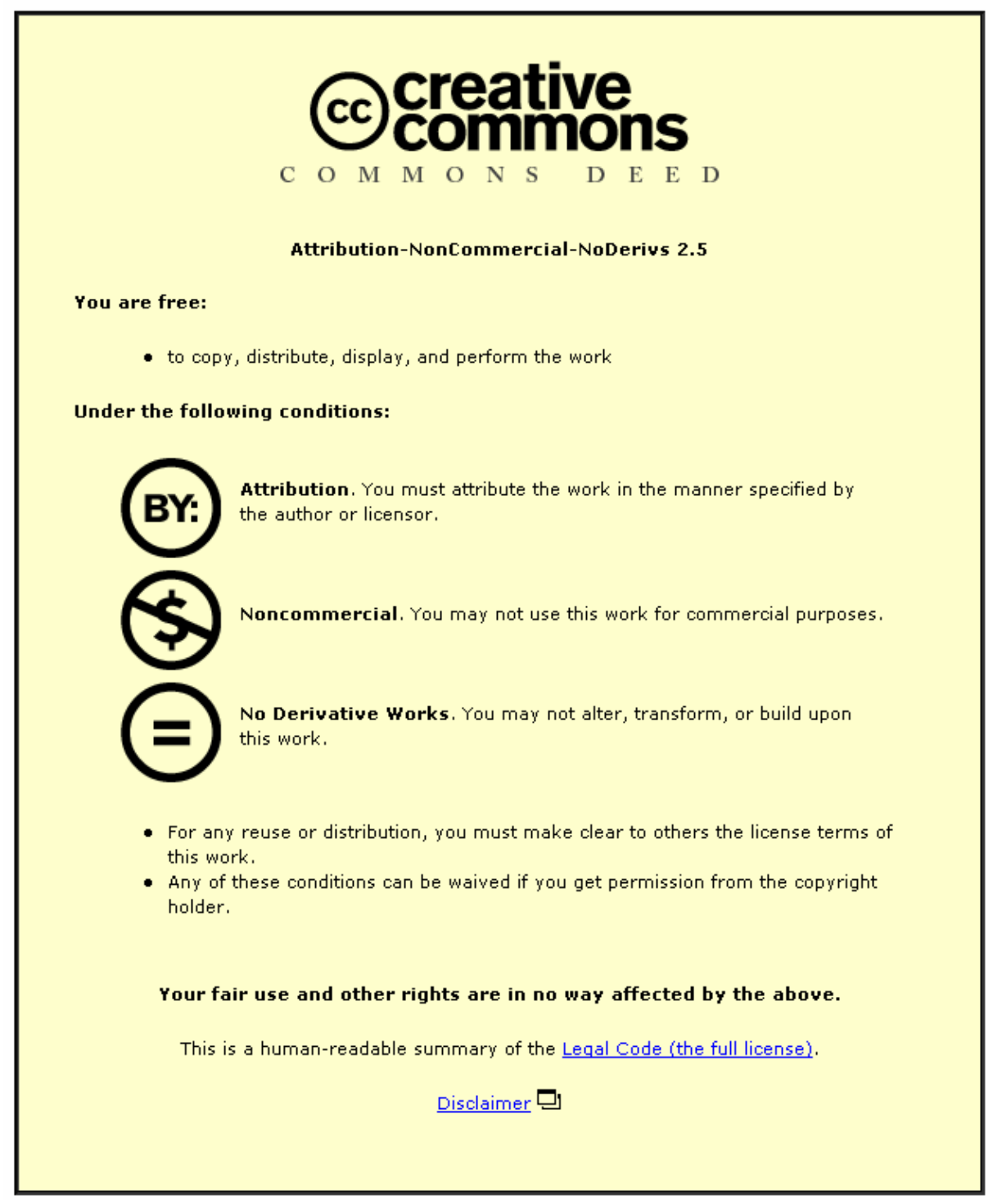

For the full text of this licence, please go to: http://creativecommons.org/licenses/by-nc-nd/2.5/ 


\title{
Influence of threshold variation on determining the properties of a polymer electrolyte fuel cell gas diffusion layer in X-ray nano-tomography
}

\author{
H. Ostadi, K. Jiang \\ Centre for Biomedical and Nanotechnology, School of Mechanical Engineering, University of \\ Birmingham, Birmingham B15 2TT, UK \\ P. Rama, Y. Liu, R. Chen
}

Department of Aeronautical and Automotive Engineering, Loughborough University, Leicestershire LE11 3TU, UK

X.X. Zhang

Department of Engineering, University of Liverpool, Brownlow Street, Liverpool L69 3GH, UK

\begin{abstract}
Morphological parameters of a 3D binary image of a porous carbon gas diffusion layer (GDL) for polymer electrolyte fuel cells (PEFC) reconstructed using X-ray nano-tomography scanning have been obtained, and influence of small alterations in the threshold value on the simulated flow properties of the reconstructed GDL has been determined. A range of threshold values with $0.4 \%$ increments on the greyscale map have been applied and the gas permeability of the binary images have been calculated using a single-phase lattice Botlzmann model (LBM), which is based on the treatment of nineteen velocities in the three dimensional domain (D3Q19). The porosity, degrees of anisotropy and the mean pore radius have been calculated directly from segmented voxel representation. A strong relationship between these parameters and threshold variation has been established. These findings suggest that threshold selection can significantly affect some of the flow properties and may strongly influence the computational simulation of micro and nano-scale flows in a porous structure.
\end{abstract}


Keywords: X-ray nano-tomography; Three-dimensional reconstruction; Threshold; Lattice Boltzmann modelling; Gas diffusion layer; Micro-structure porous materials 


\section{Introduction}

X-ray nano-computed tomography (nano-CT) enables non-invasive $3 \mathrm{D}$ visualisation and characterisation of both external and internal structures of samples, for example integrated circuit (IC) chips or porous materials such as the fuel cell GDL. In nano-CT several hundred 2D images of a physical sample over a range of angles are taken and the level of X-ray attenuation in the images according to the density of the sample is mapped. These shadow images are in 256 shades of grey and can be compiled to a stack and another stack of $2 \mathrm{D}$ images are created corresponding to what would be seen if the sample is cut through the scanning plane. The slices can then be assembled to reconstruct a 3D digital model (Imbaby et al.; Carmignato et al., 2009; Zschech et al., 2008).

The two types of high resolution X-ray tomography scanners currently available are micro-tomography and nano-tomography systems. The former provides a resolution of $1-10 \mu \mathrm{m}$, while the latter can produce a resolution of less than $100 \mathrm{~nm}$. In addition, scanning transmission X-ray microscopes (STXMs) operating at the National Synchrotron Light Source and Advanced Photon Source have been used for nano-tomography. The latest laboratory-based X-ray nano-tomography can achieve a resolution of $400 \mathrm{~nm}$ with detectability of 100-200 nm (McNulty et al., 1995; Warwick et al., 1998; Cai

et al., 2000; Parkinson and Sasov, 2008). Recently, X-raymicro-tomography has been used to quantify liquid water saturation distribution in porous GDLs and determining the Q1 two-phase material parameters with a sub $10 \mathrm{~mm}$ resolution (Becker et al., 2008; Sinha et al., 2006). The latest report suggests that nano-tomography (Nano XCT, US: Xradia Corp.) can be used to reconstruct the porous catalyst layer $(\mathrm{CL})$ of fuel cells at sub-micron resolution in order to study the changes in membranes after transient operation (Garzon et al., 2007). Nano-tomography has also been successfully employed to scan porous human dentin tubules at $295 \mathrm{~nm}$ resolution and to reconstruct the 3D cylindrical structure of the porous tubules, which are between 1 and $3 \mu \mathrm{m}$ in diameter using the SkyScan desktop nano-tomography system (SkyScan 2011, Belgium: SkyScan) (Parkinson and Sasov, 2008).

Computer modelling of porous materials such as GDL is used extensively in flow simulation and characterisation. These porous materials have to be purposefully designed to allow reactant gases to pass through to the $C L$ while removing excess product water (Parkinson and Sasov, 2008; Becker et al., 2008; Sinha et al., 2006; Garzon et al., 2007; Barbir, 2005; Sinha and Wang, 2007; Sinha et al., 
2007). The transport phenomena inside a GDL are dependent on the local micro-structural characteristics of the pore network (Thiedmann et al., 2009 R. Thiedmann, C. Hartnig, I. Manke, V. Schmidt and W. Lehnert, Local structural characteristics of pore space in GDLs of PEM fuel cells based on geometric 3D graphs, J. Electrochem. Soc. 156 (11) (2009), pp. B1339-B1347. Full Text via CrossRef | View Record in Scopus | Cited By in Scopus (11) Thiedmann et al., 2009). For fuel cell flow simulation in a porous layer and pore analysis, representative binary models are required which define the internal solid-void boundaries in a GDL structure. Therefore, it is necessary to threshold the greyscale images acquired from X-ray tomography in order to produce binary images (Manke et al., 2007; Feser et al., 2006; Hung et al., 2008).

One of the crucial steps in the X-ray tomography based digital reconstruction process is thresholding, in which structures are evaluated in voxels with grey level values and segmented to distinguish solids from pores using a threshold value. At present, a threshold is often determined visually or by an algorithm supplied by the manufacturer of the scanning system. However, it has been shown that the selection of threshold is crucial and can have a significant effect on the volume fractions, pore sizes, trabecular thickness, morphological parameters and mechanical properties of various structures (Imbaby et al.; Ostadi et al., 2008; Ding et al., 1999; Hara et al., 2002; Thiedmann et al., 2008). As such, further research is needed to improve the understanding of how optimal threshold levels can be determined to enhance the accuracy and practical applicability of X-ray tomography based structural modelling and porous flow simulation.

The purpose of this study is to understand the effect of threshold variation on the properties of reconstructed porous materials. A GDL sample of 5-10 $\mu \mathrm{m}$ filament size has been scanned in a SkyScan nano-tomography scanner (SkyScan 2011, Belgium: SkyScan) with a pixel resolution of 680 $\mathrm{nm}$. Next, a set of 2D greyscale image slices were produced in 256 greyscales and thresholded in 7 grey levels with a step of $0.4 \%$ variation, such that each step equates to 1 grey level. This process results in a seven sets of thresolded $2 \mathrm{D}$ image slices. The key structural parameters were calculated directly from seven 3D digital models, which were constructed with the seven corresponding sets of image slices. Afterwards, well-tested LB model (Bals et al., 2007; Zhang et al., 2002; Zhang and Ren, 2003; Wang et al., 2005) was applied to simulate single-phase flows through each of these 3D digital models. Finally, the resulting permeability and its sensitivity to the threshold variation were analysed. 


\section{Experimental}

In this investigation, a $1 \times 1 \times 0.3 \mathrm{~mm}$ carbon paper GDL sample without a microporous layer (Technical Fibre Products Ltd., UK) was scanned in front of an X-ray source of SkyScan 2011 with $25 \mathrm{kV}, 200 \mu \mathrm{A}$ without filter, $2 \mathrm{~s}$ exposure time, 3 frames in average and a rotation step of 0.5 degrees. 371 shadow images with $680 \mathrm{~nm}$ pixel resolution were acquired within $40 \mathrm{~min}$. The shadow images were then processed using modified Feldkamp filtered back projection method to reconstruct the 2D greyscale slices (SkyScan Microtomography, 2007). CTAN software (CT-analyzer, Belgium) was then employed to reconstruct a 3D digital model of the sample, as shown in Figs. 1a (SkyScan Microtomography, 2007). Figs. $1 \mathrm{~b}$ and $\mathrm{c}$ show a typical shadow and a reconstructed cross-section image of a carbon paper GDL. Fig. 1d is a typical binary image of Fig. 1c that is used for calculations.

3D and 2D images of a GDL carbon paper sample. (a) An isometric view of the reconstructed image of GDL sample with $680 \mathrm{~nm}$ pixel resolution using CTAN, (b) a shadow X-ray tomographic image, (c) a 2D reconstructed slice image, and (d) a binary image of the cross-section shown in Fig. 1d.

\section{Calculations of structural parameters}

A key parameter to determine the capacity of gas and water transport in GDL is its porosity $\varepsilon$ defined by the ratio of pore volume over total volume. A GDL should be sufficiently porous to allow the flow of reactant gas and water, be electrically and thermally conductive, and have adequate rigidity in order to support the membrane electrolyte assembly. The calculation of porosity can be summarised in two steps. (1) A threshold is applied to the reconstructed grey scale images and the binary images are thus obtained. In each binary image 0 and 1 represent void space and solid space, respectively. (2) A cubic matrix of these images is built and the ratio between the number of zeros and the total number of elements in the matrix is obtained as the porosity of the GDL layer.

The anisotropy in porous media properties was found to be the most important factor of mechanical strength (Odgaard, 1997). Isotropy (or anisotropy) is a measure of 3D symmetry or the presence (or absence) of preferential alignment of structures along a particular directional axis. In a complexly structured volume containing void and solid, if the volume is isotropic, then a straight line passing through the volume at any orientation will make a similar number of intercepts through the solid phase. A GDL could be anisotropic, since lines going along the direction of filaments would make few 
intercepts while lines crossing at right angle to the direction of the majority of the filaments would make many intercepts (Fig. 1a). The mean intercept length (MIL) measures isotropy by sending a line through a 3D image containing binary objects and then by dividing the length of the test line through the analysed volume by the number of intercepts between the lines and the solids. A large number of test lines are drawn passing through each point of the volume and anisotropy tensor analysis is used. Finally, one can derive a single parameter from the tensor eigenanalysis measuring anisotropy and express it as the minimum eigenvalue divided by the maximum eigenvalue. Degrees of anisotropy calculated in this way vary from 0 (fully isotropic) to 1 (fully anisotropic) (SkyScan Microtomography., 2007).

The mean pore radius is an important parameter in GDL characterisation as Knudson diffusion coefficient is a linear function of mean pore radius (Weber and Newman, 2004). In the analysis of 3D images produced using nano-CT, a 3D spherical local radius can be measured (Ulrich et al., 1999). The local radius for a point in a solid/pore is defined as the radius of a sphere with two conditions (Hildebrand and Ruegsegger, 1997): (1) the sphere encloses the point and (2) the sphere is entirely bounded within the solid/pore surfaces.

The above parameters have been calculated directly from the voxel representation through CTAN software based on a sample volume of $300 \times 700 \times 900 \mu \mathrm{m}$ with $680 \mathrm{~nm}$ pixel resolution.

\section{Calculations of fluid transport parameter}

Permeability is one of the most important transport parameters of a porous material, describing the ability of the medium to conduct viscous fluids. The permeability of a medium depends on its pore geometry. Simulating gas flow through the void space in the 3D X-ray image can reveal the velocity field in detail and help estimate the permeability. However, the complexity of the 3D pore geometry in the GDL makes a direct simulation of fluid flow through it difficult. In this research the LB model was used to simulate the microscopic velocity field and thus the absolute permeability as a macroscopic property.

Unlike the traditional computational fluid dynamics (CFD) the LB method does not solve a group of partial differential equations, but simulates fluid flow by tracking the movement and collisions of a number of fictitious fluid particles in lattice (Chen et al., 1992; McNamara and Zanetti, 1988; Frisch et 
al., 1986; He and Luo, 1997).The following equation describes the movement and collisions of the fluid particles:

$f_{i}\left(x+e_{i} \delta t, t+\delta t\right)=f_{i}(x, t)+\left[f_{i}^{e q}(x, t)-f_{i}(x, t)\right] / \pi$

where $f_{i}(x, t)$ is the distribution function of the particle at location $x$ and time $t ; e_{i}$ the velocity, $f_{i}^{e q}(x, t)$ the value of $f_{i}(x, t)$ at equilibrium, and $\tau$ is a dimensionless relaxation parameter that controls the rate at which $f_{i}(x, t)$ approaches $f_{i}^{e q}(x, t)$. The particles in each void voxel are allowed to move in 19 directions from the origin, as shown in Fig. 2, which includes stagnation at the origin, $e_{0}$.

The equilibrium distribution of particles moving in each of the 19 direction is given by

$f_{i}^{e q}(x, t)=w_{i}\left\{\rho+\rho_{0}\left[\frac{1}{c_{s}^{2}}\left(e_{i} u\right)+\frac{1}{2 c_{s}^{4}}\left(e_{i} u\right)^{2}-\frac{1}{2 c_{s}^{2}}(u u)\right]\right\}$

where $w_{i}$ is a weighting factor in which $w_{i}=1 / 3$ for $\left|e_{i}\right|=0, w_{i}=1 / 18$ for $\left|e_{i}\right|=\delta x / \delta t$ and $w_{i}=1 / 36$ for $\left|e_{i}\right|=\sqrt{2} \delta x / \delta t, \rho$ is fluid density, $\rho_{0}$ is a reference fluid density, $u$ is the bulk fluid velocity, $\delta x$ is the side length of the voxel and $\delta t$ is a time step. The density and the bulk fluid velocity in each void voxel are calculated from

$\rho(x, t)=\sum_{i=1}^{8} f_{i}(x, t)=\sum_{i=1}^{8} f_{i}^{e q}(x, t)$

$\rho_{0} u(x, t)=\sum_{i=1}^{8} e_{i} f_{i}(x, t)=\sum_{i=1}^{8} e_{i} f_{i}^{e q}(x, t)$

The above LB framework simulates the dynamics of a fluid with kinematic viscosity $v$ given by $v=\delta x^{2}(\tau-0.5) / 3 \delta t$ and pressure by $P=c_{s}^{2} \rho$. Implementation of the LB model involves a collision step and a streaming step during each time step $\delta t$. The collision step calculates the term on the lefthand side of Eq. (1) as $f_{i}^{*}(x, t)=f_{i}(x, t)+\left[f_{i}^{e q}(x, t)-f_{i}(x, t)-f_{i}(x, t)\right] / \tau$, and the streaming step moves the pro-collision result from the voxel at $x$ to its adjacent voxel at $x+e_{i} \delta t$; the time for the particle to complete such a movement is $\delta t$; that is $f_{i}^{*}\left(x+e_{i} \delta t, t+\delta t\right)=f_{i}^{*}(x, t)$.

To simulate gas flow, a pressure gradient is imposed in one direction (the $\mathrm{x}$ direction). The four sides of the image in other two directions are treated as periodic boundaries such that any particle coming out of the image from any side re-enters the image through its opposite side without changing its momentum. All simulations start from a zero velocity field and are terminated once the flow in the void 
space is deemed to have reached steady state. If the size of the image in the $x, y$ and $z$ directions are denoted by $L_{x}, L_{y}$ and $L_{z}$, respectively, when flow reached steady state, the average flow rates over the image in the three directions are

$q_{x}=\frac{\sum_{i} u_{x}\left(x_{i}+y_{i}+z_{i}\right)}{L_{x} L_{y} L_{z}}, q_{y}=\frac{\sum_{i} u_{y}\left(x_{i}+y_{i}+z_{i}\right)}{L_{x} L_{y} L_{z}}, q_{z}=\frac{\sum_{i} u_{z}\left(x_{i}+y_{i}+z_{i}\right)}{L_{x} L_{y} L_{z}}$

where $u_{x}\left(x_{i}+y_{i}+z_{i}\right), u_{y}\left(x_{i}+y_{i}+z_{i}\right)$, and $u_{z}\left(x_{i}+y_{i}+z_{i}\right)$ are the three velocity components of fluid in the void the voxel located at $\left(x_{i}+y_{i}+z_{i}\right)$. Once the average flow rates are known, the absolute permeability of the image along and off the flow direction is calculated from the Darcy's law as follows:

$k_{x x}=\frac{\rho_{0} v q_{x}}{\left(\Delta P / L_{x}\right)}, k_{x y}=\frac{\rho_{0} v q_{y}}{\left(\Delta P / L_{x}\right)}, k_{x z}=\frac{\rho_{0} v q_{z}}{\left(\Delta P / L_{x}\right)}$

where $\Delta P$ is the pressure drop applied at the two opposite sides of the images in the $x$ direction. Applying the pressure drop in the $y$ and $z$ directions allows obtaining the permeability in other two directions.

The LBE model was applied to a sample of $100 \times 300 \times 100 \mu \mathrm{m}^{3}$ (where the $300 \mu \mathrm{m}$ length corresponds to the principal flow direction) to simulate the gas flow through the void space in the GDL.

\section{Image processing and results}

Images from SkyScan 2011 nano-tomography scanner are in 256 greyscales. Thresholding process defines a partition on the greyscale, where darker regions in the $2 \mathrm{D}$ image slices represent solid space, while lighter regions represent void space. For instance, if an operator chooses 100 to be the threshold value, then pixels with grey levels less than 100 will be taken as void while pixels with grey value greater than 100 will be taken as solid.

In this research, a reference threshold value was chosen for processing greyscale images and creating binary images. Six more threshold values in increments of 1 on the greyscale were subsequently used as test threshold values and the corresponding binary images were generated.

\subsection{Threshold variation and structural parameter}


The first important parameter that can be calculated directly from a reconstructed model is porosity using the method described in Section 3. Fig. 3a shows that the porosity of a $300 \times 700 \times 900 \mu m^{3}$ region, which is the full area of tomography image and shows that the porosity changes linearly over the range of thresholds for the seven samples.

Since the calculation of degrees of anisotropy is time consuming, a cubic sample of $300 \mu \mathrm{m}$ in length (equalling the thickness of the carbon paper) was selected for calculation purposes. Dimensionless degrees of anisotropy of the sample over the threshold variation is summarised in Fig. 3b for both pore and solid networks. It can be seen that threshold variation has a little effect on the degree of anisotropy for both solid and pore network in the GDL. A 3\% variation in threshold results in a $2 \%$ change in the degree of anisotropy.

The average pore size of the 7 samples measuring $300 \times 700 \times 900 \mu \mathrm{m}$ has been calculated and was found to change with the threshold value by 0.5 while the average pore diameter increased by $25 \%$ from 13.5 to $16.9 \mu \mathrm{m}$ over a $3 \%$ threshold variation span linearly. This means that Knudson diffusion coefficient may also change about $25 \%$ over only $3 \%$ of threshold variation. The variation of the average pore diameter over threshold is shown in Fig. 4.

\subsection{Threshold variation and fluid transport parameter}

The reconstructed 3D model of the GDL is stored in a 3D binary array and uploaded to the LB model for porous flow simulation. In general modelling terms, the spatial resolution of the LB model has to match the pixel resolution of the 3D image. In the current study the GDL has a porosity of greater than $80 \%$, which thereby eases the computational demand of the LB flow simulation. This therefore allows each voxel of the 3D binary image to be used directly as the lattices of the LB model. In this case, the spatial resolution of the LB model is set equal to the pixel resolution of the X-ray images. The application of the LB model to simulate the permeability of the carbon paper GDL was validated by the authors in previous work (Rama et al., in press).

The LB model was applied to four differently thresholded samples of $100 \times 300 \times 100 \mu \mathrm{m}$ flowing along $300 \mu \mathrm{m}$ length direction to simulate the detailed gas velocity field in the void space of the GDL, with the assumption that the void spaces were filled with air. The simulated velocity was then used to obtain the absolute permeability of the region. Because absolute permeability represents linear 
dependence of gas flow rate on pressure gradient, it should be ensured that the flow rate in the simulations is also in this linear range. As such, the pressure difference applied to each region was set to $20 \mathrm{~Pa}$. The simulations were carried out on a dual-core $2.01 \mathrm{GHz}$ workstation with $3.25 \mathrm{~GB}$ RAM. A single-phase simulation for the region took $500 \mathrm{~min}$. Fig. 5 illustrates that the through-plane permeability $\left(K_{x x}\right)$ is a strong function of threshold variation.

\section{Conclusions}

In reconstruction of a digital model of a porous material from X-ray nano-tomography scans, a small variation in threshold may have a significant influence on structural properties of the structural model and on its simulated flow parameters. The aim of this study was to quantify the significance of the influence of the threshold variation to the key parameters of porous materials for the first time. The outcomes of this research provide a useful guidance to researchers in the selection of adequate threshold levels in a variety of tomography-based techniques.

In this research, the influence of a small threshold variation on the mechanical and fluidic flow properties of a carbon fibre GDL layer was studied. The results show that porosity increases linearly with reducing threshold. The linearity implies that the GDL structure can be assumed to be generally homogeneous in terms of material density distribution. The sensitivity of the degree of anisotropy for the solid/pore network to a $3 \%$ threshold variation is only about $2 \%$.

The analysis on average pore diameter finds that it increases from 13.5 to $16.9 \mu \mathrm{m}$ and can potentially therefore have an influence of $25 \%$ on Knudson diffusion coefficient of the GDL. Hence diffusion is a parameter sensitive to threshold.

Permeability is found very sensitive to the threshold and changes about $85 \%$ with only $3 \%$ threshold variation since increasing the porosity helps fluidic flow in the pore network. One way to reduce the effect of threshold on permeability is using a higher resolution scanner.

In conclusion, a small variation in the selection of threshold has a great effect on permeability, porosity and Knudson diffusion coefficient and negligible effect on degree of anisotropy. Researchers should be aware of the significance of selecting a threshold value towards the final simulation results when they process reconstructed images obtained from X-ray nano-tomography on porous materials. 


\section{Acknowledgments}

This research is supported by the UK Technology Strategy Board (TSB Project no.: TP/6/S/K3032 H). We acknowledge the kind support from industrial partners AVL List GmbH, Intelligent Energy Ltd., Johnson Matthey Fuel Cells Ltd., Saati Group Inc. and Technical Fibre Products Ltd.

\section{References}

Bals et al., 2007 S. Bals, K.J. Batenburg, J. Verbeeck, J. Sijbers and G.V. Tendeloo, Quantitative 3D reconstruction of catalyst particles for bamboo-like carbon-nanotubes, Nano Lett. 7 (12) (2007), pp. 3669-3674. Full Text via CrossRef | View Record in Scopus | Cited By in Scopus (17)

Barbir, 2005 F. Barbir, PEM Fuel Cell: Theory and Practice, Elsevier Academic Press, Burlington, Massachusetts (2005) 93.

Becker et al., 2008 J. Becker, V. Schulz and A. Wiegmann, Numerical determination of two-phase material parameters of a gas diffusion layer using tomography images, J. Fuel Cell Sci. Technol. 5 (2) (2008), pp. 021006-1-021006-9.

Cai et al., 2000 Z. Cai, B. Lai, W. Yun, I. McNulty, A. Khounsary, J. Maser, P. Ilinski, D. Legnini, E. Trakhtenberg, S. Xu, B. Tieman, G. Wiemerslage and E. Gluskin, Performance of a high-resolution Xray microprobe at the advanced photon source, AIP Conf. Proc. 521 (2000), pp. 31-34. Full Text via CrossRef

Carmignato et al., 2009 S. Carmignato, D. Dreossi, L. Mancini, F. Marinello, G. Tromba and E. Savio, Testing of X-ray microtomography systems using a traceable geometrical standard, Meas. Sci. Technol. 20 (2009), pp. 084021-084028. Full Text via CrossRef | View Record in Scopus | Cited By in Scopus (5)

Chen et al., 1992 H. Chen, S. Chen and W.H. Matthaeus, Recovery of the Navier-Stokes equations using a lattice-gas Boltzmann method, Phys. Rev. A 45 (1992), pp. R533-R5342.

Ding et al., 1999 M. Ding, A. Odgaard and I. Hvid, Accuracy of cancellous bone volume fraction measured by micro-CT scanning, J. Biomech. 32 (1999), pp. 323-326. Article | PDF (272 K) | View Record in Scopus | Cited By in Scopus (84) 
Feser et al., 2006 J.P. Feser, A.K. Prasad and S.G. Advani, Experimental characterization of in-plane permeability of gas diffusion layers, J. Power Sources 162 (2) (2006), pp. 1226-1231. Article | PDF (578 K) | View Record in Scopus | Cited By in Scopus (58)

Frisch et al., 1986 U. Frisch, B. Hasslacher and Y. Pomeau, Lattice-gas automata for the NavierStokes equation, Phys. Rev. Lett. 56 (1986), pp. 1505-1508. Full Text via CrossRef | View Record in Scopus | Cited By in Scopus (853)

Garzon et al., 2007 F.H. Garzon, S.H. Lau, J.R. Davey and R.L. Borup, Micro and nano X-ray tomography of PEM fuel cell membrane after transient operation, ECS Trans. Proton Exch. Membr. Fuel Cells 11 (1) (2007), pp. 1139-1150.

Hara et al., 2002 T. Hara, E. Tanck, J. Homminga and R. Huiskes, The influence of microcomputed tomography threshold variations on the assessment of structural and mechanical trabecular bone properties, Bone 23 (2) (2002), pp. 163-169.

He and Luo, 1997 X. He and L. Luo, Theory of the lattice Boltzmann method: from the Boltzmann equation to the lattice Boltzmann equation, Phys. Rev. E 55 (1997), pp. 6333-6336.

Hildebrand and Ruegsegger, 1997 T. Hildebrand and P. Ruegsegger, A new method for the modelindependent assessment of thickness in three-dimensional images, J. Microsc. (Oxford) 185 (1997), pp. 67-75. View Record in Scopus | Cited By in Scopus (439)

Hung et al., 2008 T.F. Hung, J. Huang, H.J. Chuang, S.H. Bai, Y.J. Lai and Y.W. Chen-Yang, highly efficient single-layer gas diffusion layers for the proton exchange membrane fuel cell, J. Power Sources 184 (1) (2008), pp. 165-171. Article | PDF (1270 K) | View Record in Scopus | Cited By in Scopus (11)

Imbaby et al., 2009 Imbaby, M., Ostadi, H., Jiang, K., 2009. Characterisation of stainless steel microparts fabricated by soft moulding technique, Micro. Nano Lett. 4(2), 99-105.

Manke et al., 2007 I. Manke, C. Hartnig, M. Grunerbel, W. Lehnert, N. Kardjilov, A. Haibel, A. Hilger and J. Banahrt, Investigation of water evolution and transport in fuel cells with high resolution synchrotron X-ray radiography, Appl. Phys. Lett. 90 (17) (2007), pp. 4501-4505. 
McNamara and Zanetti, 1988 G.R. McNamara and G. Zanetti, Use of the Boltzmann-equation to simulate lattice-gas automata, Phys. Rev. Lett. 61 (20) (1988), pp. 2332-2335. Full Text via CrossRef | View Record in Scopus | Cited By in Scopus (670)

McNulty et al., 1995 I. McNulty, W.S. Haddad, J.E. Trebes and E.H. Anderson, Soft X-ray scanning microtomography with submicrometer resolution, Rev. Sci. Instrum. 66 (1995), pp. 1431-1433. Full Text via CrossRef | View Record in Scopus | Cited By in Scopus (11)

Odgaard, 1997 A. Odgaard, Three-dimensional methods for quantification of cancellous bone architecture, Bone 20 (6) (1997), pp. 315-328. Article | PDF (1726 K) | View Record in Scopus | Cited By in Scopus (290)

Ostadi et al., 2008 H. Ostadi, K. Jiang and P.D. Prewett, Micro/Nano X-ray tomography reconstruction fine-tuning using scanning electron microscope images, Micro Nano Lett. 3 (4) (2008), pp. 106-109. Full Text via CrossRef | View Record in Scopus | Cited By in Scopus (7)

Parkinson and Sasov, 2008 C.R. Parkinson and A. Sasov, High-resolution non-destructive 3D interrogation of dentin using X-ray nanotomography, Dent. Mater. 24 (2008), pp. 773-777. Article | PDF (591 K) | View Record in Scopus | Cited By in Scopus (9)

Rama et al., 2010 Rama, P., Liu, Y., Chen, R., Ostadi, H., Jiang, K., Zhang, X., Fisher, R., Jeschke, M., An X-ray tomography based Lattice Boltzmann simulation study on gas diffusion layers of polymer electrolyte fuel cells, J. Fuel. Cell Sci. Technol. (in press).

Sinha and Wang, 2007 P.K. Sinha and C.Y. Wang, Pore-network modeling of liquid water transport in gas diffusion layer of a polymer electrolyte fuel cell, Electrochim. Acta 52 (28) (2007), pp. 7936-7945. Article | PDF (1811 K) | View Record in Scopus | Cited By in Scopus (79)

Sinha et al., 2006 P.K. Sinha, P. Halleck and C.Y. Wang, Quantification of liquid water saturation in PEM fuel cell diffusion medium using X-ray microtomography, Electrochem. Solid State 9 (7) (2006), pp. A344-A348. Full Text via CrossRef | View Record in Scopus | Cited By in Scopus (93) 
Sinha et al., 2007 P.K. Sinha, P.P. Mukherejee and C.Y. Wang, Impact of GDL structure and wettability on water management in polymer electrolyte fuel cells, J. Mater. Chem. 17 (30) (2007), pp. 3089-3103. View Record in Scopus | Cited By in Scopus (65)

SkyScan Microtomography., 2007 SkyScan Microtomography, 2007. CT-Analyser Users Guide, version 1.6.1, Kartuizersweg, Belgium.

Thiedmann et al., 2008 R. Thiedmann, F. Fleischer, C. Hartnig, W. Lehnert and V. Schmidt, Stochastic 3D modeling of the GDL structure in PEMFCs based on thin section detection, J. Electrochem. Soc. 155 (4) (2008), pp. B391-B399. Full Text via CrossRef | View Record in Scopus | Cited By in Scopus (10)

Thiedmann et al., 2009 R. Thiedmann, C. Hartnig, I. Manke, V. Schmidt and W. Lehnert, Local structural characteristics of pore space in GDLs of PEM fuel cells based on geometric 3D graphs, J. Electrochem. Soc. 156 (11) (2009), pp. B1339-B1347. Full Text via CrossRef | View Record in Scopus | Cited By in Scopus (11)

Ulrich et al., 1999 D. Ulrich, B. van Rietbergen, A. Laib and P. Rüegsegger, The ability of threedimensional structural indices to reflect mechanical aspects of trabecular bone, Bone 25 (1) (1999), pp. 55-60. Article | PDF (91 K) | View Record in Scopus | Cited By in Scopus (261)

Wang et al., 2005 J. Wang, X. Zhang, A.G. Bengough and G.W. Crawford, Domain-decomposition method for parallel lattice Boltzmann simulation of incompressible flow in porous media, Phys. Rev. E 72 (2005), pp. 0167061-01670611.

Warwick et al., 1998 T. Warwick, K. Franck, J.B. Kortright, G. Meigs, M. Moronne, S. Myneni, E. Rotenberg, S. Seal, W.F. Steele, H. Ade, A. Garcia and S. Cerasari, A scanning transmission X-ray microscope for materials science spectromicroscopy at the advanced light source, Rev. Sci. Instrum. 69 (1998), pp. 2964-2967. OJPS full text | Full Text via CrossRef

Weber and Newman, 2004 A.Z. Weber and J. Newman, Modeling transport in polymer-electrolyte fuel cells, Chem. Rev. 104 (2004), pp. 4679-4726. Full Text via CrossRef | View Record in Scopus | Cited By in Scopus (219) 
Zhang and Ren, 2003 X. Zhang and L. Ren, Lattice Boltzmann model for agrochemical transport in soils, J. Contam. Hydrol. 67 (2003), pp. 27-42. Article | PDF (194 K) | View Record in Scopus | Cited By in Scopus (16)

Zhang et al., 2002 X. Zhang, J.W. Crawford, A.G. Bengough and I.M. Young, On boundary conditions in the lattice Boltzmann model for advection and anisotropic dispersion equation, Adv. Water Resour. 25 (2002), pp. 601-609. Article | PDF (249 K) | View Record in Scopus | Cited By in Scopus (21)

Zschech et al., 2008 E. Zschech, W. Yun and G. Schneider, High-resolution X-ray imaging-a powerful nondestructive technique for applications in semiconductor industry, Appl. Phys. A-Mater. 92 (2008), pp. 423-429. Full Text via CrossRef | View Record in Scopus | Cited By in Scopus (6) 
a

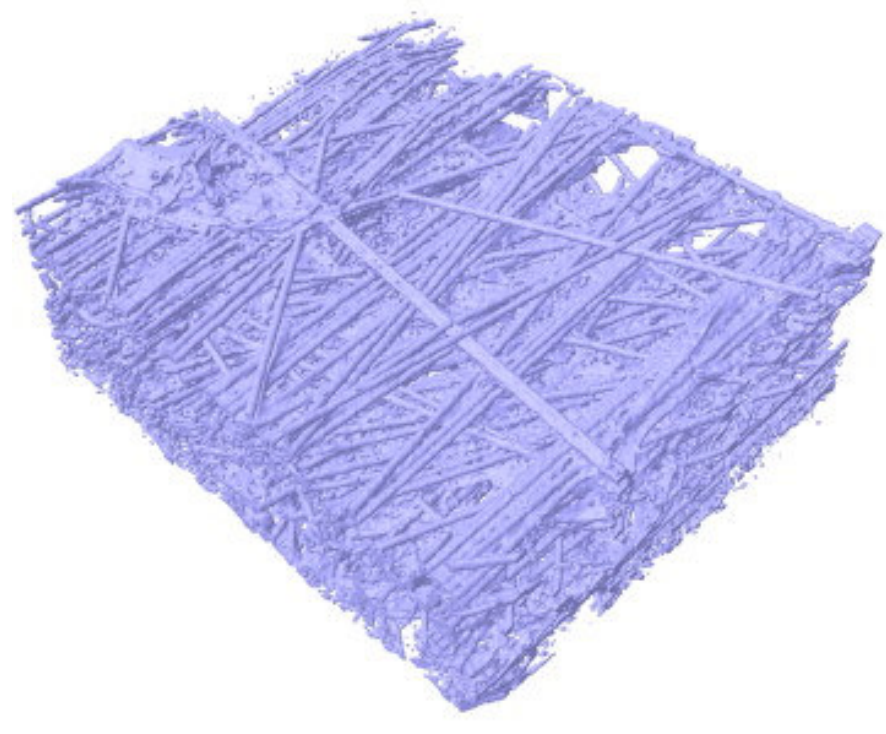

b

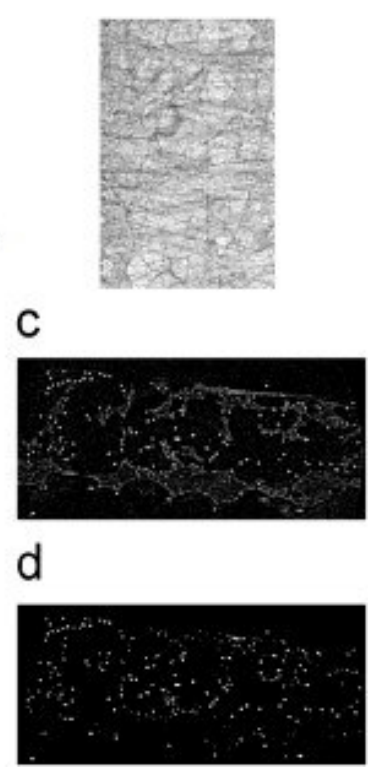

Fig. 1. 3D and 2D images of a GDL carbon paper sample. (a) An isometric view of the reconstructed image of GDL sample with $680 \mathrm{~nm}$ pixel resolution using CTAN, (b) a shadow X-ray tomographic image, (c) a 2D reconstructed slice image, and (d) a binary image of the cross-section shown in Fig. $1 d$.

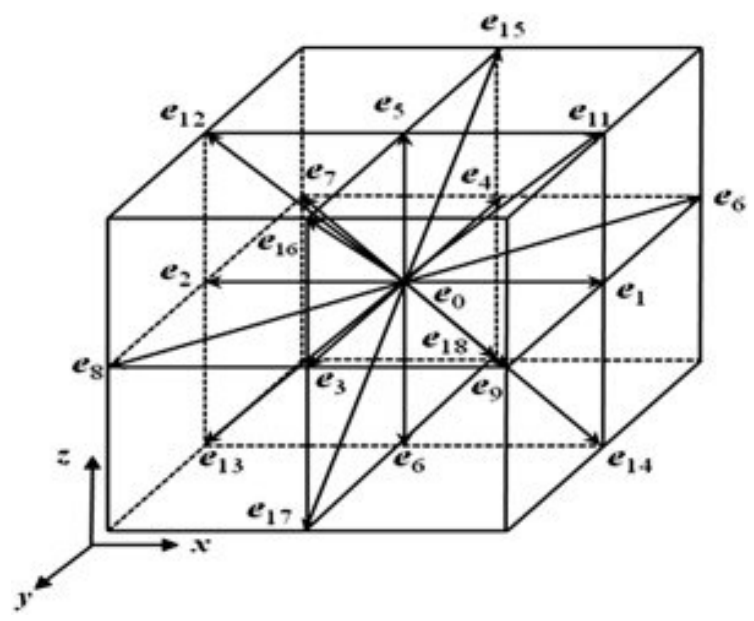

Fig. 2. The 19 directions along which the particles in each void voxel move. 

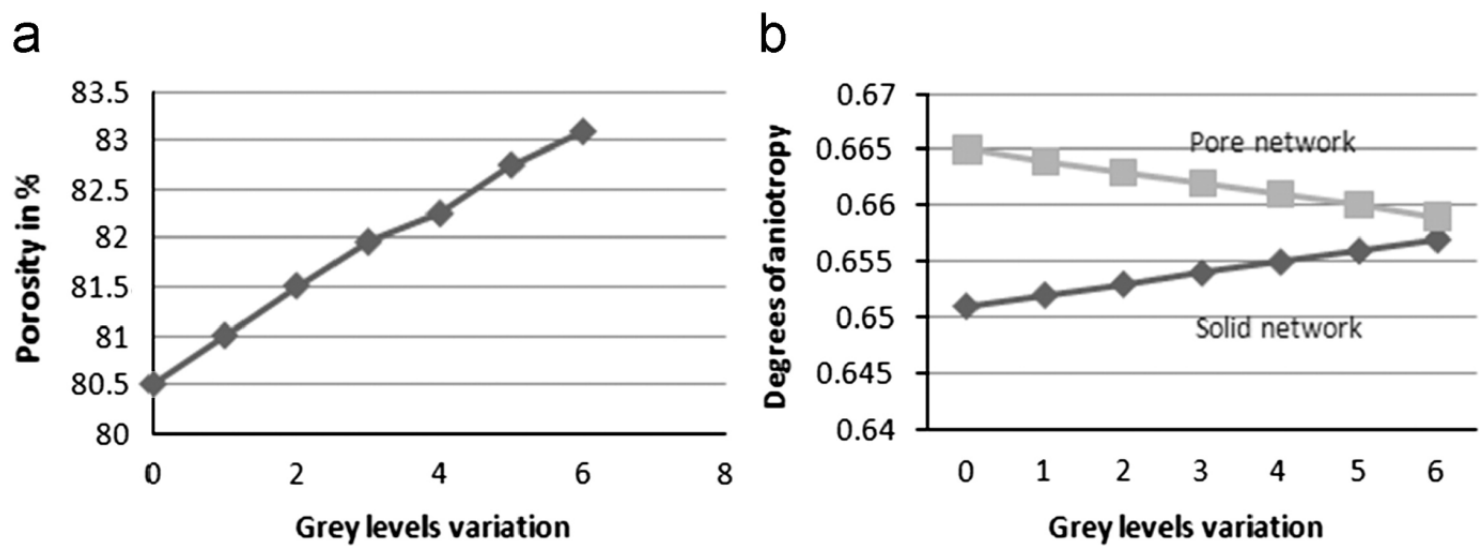

Fig. 3. Influence of threshold values on porosity and degrees of anisotropy of the GDL. (a) Porosity variation over threshold values and (b) degrees of anisotropy of both pore and solid network over threshold values.

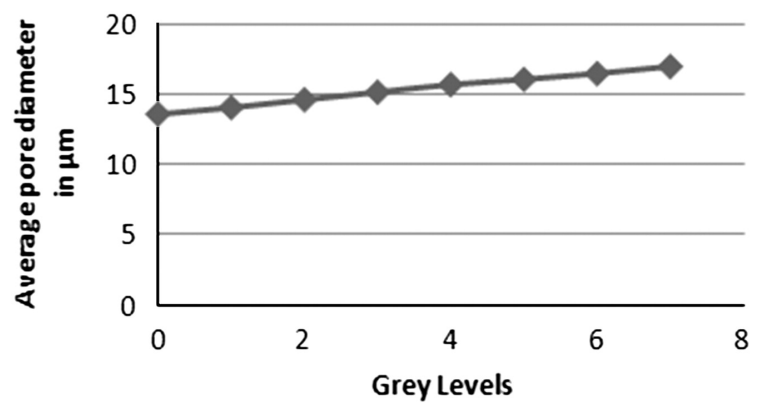

Fig. 4. The relationship between the average pore diameter of the GDL and threshold values. 


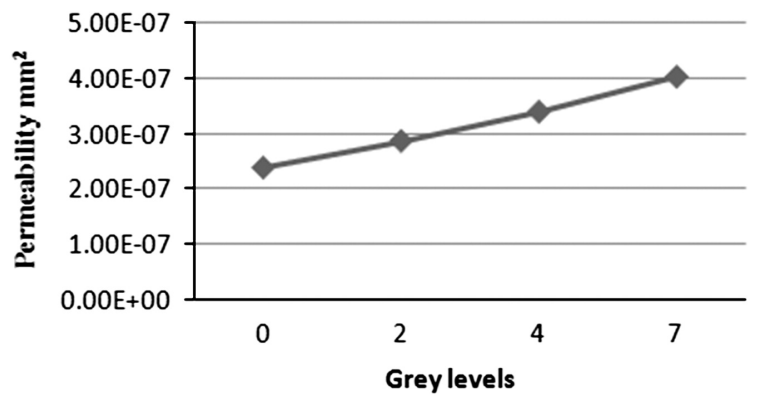

Fig. 5. Through plane permeability $\left(K_{x x}\right)$ sensitivity over threshold variation. With only $3 \%$ variation of threshold the permeability is almost doubled from $2.38 \times 10^{-7}$ to $4.02 \times 10^{-7} \mathrm{~mm}^{2}$ which is a significant change and is likely to affect the simulation results of the fuel cell. 